\title{
Review of: "Quantifying biological carbon pump pathways with a data-constrained mechanistic model ensemble approach"
}

\author{
Anna Belcher ${ }^{1}$ \\ 1 British Antarctic Survey
}

Potential competing interests: The author(s) declared that no potential competing interests exist.

\section{Review: Quantifying biological carbon pump pathways with a data-constrained mechanistic model ensemble approach}

The manuscript by Stukel, Decima and Landry is an interesting model study to assess the pathways of the biological carbon pump. The authors use the assimilation of a vast quantity of data and make the important step of incorporating higher trophic levels to the data assimilation. They highlights the paucity of zooplankton data to constrain zooplankton parameters, which is an important finding to share with the community to help future modelling efforts.

I am not a modeller by training and thus the complexities of the model are best left to evaluate by an expert in the field. However, I make some suggestions here to help the paper be understood more clearly by the non-modelling community. See specific suggestions below.

It is very interesting that the uncertainty in parameter estimation for the model is as important a source of error for export flux as variability between regions and seasons (line 406). It would be good to bring this finding into the abstract or highlight it more, as it really shows the difficulty of modelling such complex systems.

I may have missed it, but it would be good to define explicitly export flux, as this will help ensure results are not taken out of context, and the depth at which you relate export to is clear. Please put this in the methods, so it is clear the depth you refer to (absolute or relative) when do talk about export. In relation to this please make it clear throughout the manuscript that we are talking about export, as at times it comes across that you refer to deeper fluxes in the mesopelagic/sequestration. Please make sure that there is no suggestion that $\mathrm{C}$ is sequestered once exported from the euphotic zone. As noted by the authors there are many processes setting the sequestration efficiency in the mesopelagic, and it would be very interesting to see this study progressed to delve deeper and assess mesopelagic processes. As noted below when giving conclusions about the contribution of BCP pathways it must be completely transparent as to the depth this 
relates to.

I understand the authors use of constant physical forcing as there is already a lot of complexity in the model, and they state that this is the first step in hopefully even more advanced models in the future. It would be interesting ( $\mathrm{l}$ am not sure how this could be quantified) to understand the impact of this constant physical forcing on the \% contribution of mixing to the export flux. Are the authors able to make any educated suggestions as to how this \% contribution might vary with a changing physical field?

Overall the manuscript makes an excellent contribution to the field, particularly highlighted data gaps and areas that need more attention by the community to improve model accuracy and forecasting efforts.

Specific comments:

\section{Abstract}

Line 25-26: Please make it clear that we are talking about export flux, as it otherwise a bit misleading as to what depth we are talking about when we apportion importance to different BCP pathways

\section{Introduction}

Line 40: The BCP is only one of the mechanisms for long term sequestration - this reads to me as you are referring to gravitational sinking, active transport of POC. It's not clear here if you also mean passive transport processes/physical mixing etc -which processes are you wrapping up in BCP?

Line 94: It's not clear for the reader in this sentence why might get high in accuracies in zooplankton dynamics. Could you add a few words onto the sentence to say why, e.g. because no data at higher trophic levels is assimilated?

\section{Methods}

Line 127: could you give the abbreviation used here PZ and LZ for the small and large mesozooplankton. Although you give in the table, it is helpful to the reader if on first mention of terms you give the parameter abbreviation to make the text clear and avoid having to scroll back and forth to the table.

Line 184: What is the maximum depth your model extends to?

\section{Results}

Line 75-93: please refer to tables / figure where these results are detailed

Line 517: Could you define which processes are encompassed within the term 'mixing flux'

\section{Discussion}

Lines 617:618. I am not a modeller, and this part is not so clear to me. For the paper to be more easily 
understood outside the modelling community please could you give more detail on what you mean by the ' 5 different evenly spaced parameter sets' and what the significance of this is in terms of the temporal variability shown in figure 12 .

Lines 646-663: It would be good to make it clear here that the \%'s you calculate for figure 9 for export flux, may be very different when assessing the sequestration flux /flux at depths $>1000 \mathrm{~m}$ in the mesopelagic. As you note, studies have made suggestions as to the contributions of the different pathways. It would be very interesting to develop your model further to extend deeper into the mesopelagic and assess sequestration fluxes.

Figure 11: Please state the reference depth for the gravitational flux (e.g. depth of euphotic zone) Figure 12: You do not define the colours in terms of which parameter set is represented by which colour. 